JMB

\title{
Error Rate and Specificity of Human and Murine DNA Polymerase $\eta$
}

\section{Toshiro Matsuda ${ }^{1}$ Katarzyna Bebenek ${ }^{1}$, Chikahide Masutani ${ }^{3}$ Igor B. Rogozin ${ }^{5,6}$, Fumio Hanaoka ${ }^{3,4}$ and Thomas A. Kunkel ${ }^{1,2 *}$}

${ }^{1}$ Laboratory of Molecular Genetics and

${ }^{2}$ Laboratory of Structural Biology, National Institute of Environmental Health Sciences Research Triangle Park NC 27709, USA

${ }^{3}$ Institute for Molecular and Cellular Biology, Osaka University and CREST Japan Science and Technology Corporation, 1-3 Yamada-oka Suita, Osaka 565-0871, Japan

${ }^{4}$ Institute of Physical and Chemical Research (RIKEN)

Wako-shi, Saitama 3510198, Japan

${ }^{5}$ Institute of Cytology and Genetics, Siberian Branch of Russian Academy of Sciences Novosibirsk, Russia

${ }^{6}$ National Center for Biotechnology Information NLM, National Institutes of Health, Bethesda

MD 20894, USA
We describe here the error specificity of mammalian DNA polymerase $\eta$ (pol $\eta$ ), an enzyme that performs translesion DNA synthesis and may participate in somatic hypermutation of immunoglobulin genes. Both mouse and human pol $\eta$ lack intrinsic proofreading exonuclease activity and both copy undamaged DNA inaccurately. Analysis of more than 1500 single-base substitutions by human pol $\eta$ indicates that error rates for all 12 mismatches are high and variable depending on the composition and symmetry of the mismatch and its location. pol $\eta$ also generates tandem base substitutions at an unprecedented rate, and kinetic analysis indicates that it extends a tandem double mismatch about as efficiently as other replicative enzymes extend single-base mismatches. This ability to use an aberrant primer terminus and the high rate of single and double-base substitutions support the idea that pol $\eta$ may forego strict shape complementarity in order to facilitate highly efficient lesion bypass. Relaxed discrimination is further indicated by pol $\eta$ infidelity for a wide variety of nucleotide deletion and addition errors. The nature and location of these errors suggest that some may be initiated by strand slippage, while others result from additional mechanisms.
Keywords: DNA polymerase $\eta$; replication fidelity; frameshifts; tandem mutations; mismatch extension

\section{Introduction}

Human DNA polymerase $\eta$ (pol $\eta$ ) is encoded by the POLH or XPV gene. ${ }^{1,2}$ Mutations in this gene that inactivate pol $\eta$ enhance UV-induced mutagenesis and greatly increase susceptibility to sunlight-induced skin cancer. These phenotypes, resulting from the absence of pol $\eta$, likely relate to

Abbreviations used: pol $\eta$, DNA polymerase $\eta$; pol $\mathbf{l}$, DNA polymerase i; pol $\beta$, DNA polymerase $\beta$; pol $\alpha$,

DNA polymerase $\alpha$; HIV-1 RT, HIV-1 reverse transcriptase.

E-mail address of the corresponding author: kunkel@neiehs.nih.gov its ability to copy DNA templates containing cissyn thymine-thymine dimers more efficiently than most other DNA polymerases. ${ }^{3,4}$ Structure-function studies of several of the other polymerases suggest that efficient and accurate DNA synthesis depends on correct Watson-Crick base-pair geometry. ${ }^{5}$ Consistent with the importance of correct base-pair geometry to accurate replication, most DNA polymerases rarely make mistakes when copying undamaged DNA templates; their base-substitution error rates typically range from $10^{-6}$ to $10^{-4}$. In contrast, human pol $\eta$ is less accurate, ${ }^{6}$ with an average base-substitution error rate of $3.5 \times 10^{-2}$. Consistent with this high error rate are steady-state 
kinetic data indicating that human ${ }^{6,7}$ and yeast ${ }^{8}$ pol $\eta$ have low selectivity during the initial nucleotide insertion step of a polymerization reaction. These results imply that pol $\eta$ has a relaxed requirement for correct base-pairing geometry and suggest that the function of pol $\eta$ may need to be tightly controlled in vivo to prevent potentially mutagenic DNA synthesis.

To fully appreciate this mutagenic potential and to better understand the nucleotide selectivity of a DNA polymerase capable of efficiently bypassing a bulky lesion in DNA that strongly impedes synthesis by other polymerases, we provide a comprehensive view of the error specificity of pol $\eta$ from two different sources. We show that mouse pol $\eta$ is as inaccurate as its human homolog, thus demonstrating that low-fidelity synthesis during copying of undamaged DNA is inherent to mammalian pol $\eta$. We then describe the error specificity of human pol $\eta$ in detail. The results reveal that pol $\eta$ has low selectivity at the initial nucleotide insertion step, and it is promiscuous for subsequent mismatch extension. Both properties may relate to its ability to copy cyclobutane pyrimidine dimers efficiently. pol $\eta$ is also highly inaccurate for a variety of nucleotide deletion and addition errors, with the specificity suggesting that these errors are made by more than one mechanism.

\section{Results}

\section{Fidelity of mouse pol $\boldsymbol{\eta}$}

We began this study by examining the fidelity of mouse pol $\eta$ using a forward mutation assay that scores a variety of substitution, addition and del-

$\uparrow$ Control experiments indicate that a single nucleotide misincorporated into the minus strand of M13mp2 DNA during gap-filling synthesis is expressed in E. coli with an efficiency of about $60 \%$. However, minus strand expression efficiencies are somewhat lower for DNA heteroduplexes containing larger numbers of mismatched bases (data not shown, but as an example, see Umar et al. ${ }^{32}$ ) Since lacZ mutants generated by pol $\eta$ contain many sequence changes, the observed mutant frequencies of $35 \%$ (human) and $37 \%$ (mouse) suggest that essentially every gap-filled product of the pol $\eta$ reactions originally contained mutations, and that the non-mutant (i.e. blue) plaques resulted from loss of the minus strand in E. coli. Consistent with this interpretation, no mutation was found when we sequenced DNA samples isolated from six independent blue M13mp2 plaques recovered from reactions by human pol $\eta$. Because most of the lac $Z$ mutants contain many changes, silent changes are linked to changes that yield a detectable phenotype. Thus, unlike earlier studies with higher-fidelity DNA polymerases, in this study essentially all copied nucleotides within the gap become targets for the calculation of substitution, deletion and addition error rates. The error rate is simply the number of observed mutations of any particular type (from Figures 1 and 4 ) divided by the total number of nucleotides that were sequenced in all mutants (from Table 1). etion errors during synthesis to copy a 407-nucleotide template present as a single-stranded gap in M13mp2 DNA. ${ }^{6}$ Correct polymerization produces DNA that yields blue M13 plaques upon introduction of the product DNA into an Escherichia coli $\alpha$-complementation strain and plating on indicator plates. Errors are scored as light blue or colorless plaques. Like its human homolog, ${ }^{6}$ recombinant mouse pol $\eta$ lacks $3^{\prime} \rightarrow 5^{\prime}$ exonucleolytic activity and it completely filled the 407 nucleotide gap (data not shown, but see Figure 1(a) and (b) of Matsuda et $\left.a l^{6}{ }^{6}\right)$. When the products of gap-filling synthesis were introduced into E. coli, the lacZ mutant frequency among the resulting M13 plaques was $37 \%$ (average of two independent determinations). This is much greater than the mutant frequency of DNA that had not been copied in vitro $(0.06 \%)$, indicating that the lac Z mutants were generated during gap-filling synthesis. The results with mouse pol $\eta$ were similar to the $35 \%$ lac $Z$ mutant frequency obtained for synthesis catalyzed by human pol $\eta .^{6}$ As with the human enzyme, ${ }^{6}$ the majority of lac $Z$ mutant plaques generated by mouse pol $\eta$ were colorless plaque.

To determine the nature and number of errors generated by mouse pol $\eta$, we isolated DNA from 20 independent lac $Z$ mutants and sequenced nucleotides +191 (the start of synthesis) through -84 (the $5^{\prime}$ end of the lacZ gene in M13mp2). For comparison, and to obtain a detailed view of the mutagenic potential of human pol $\eta$, we expanded our earlier analysis of the error specificity of human pol $\eta^{6}$ by sequencing all 407 nucleotides in the gap (+191 through -216) in 124 independent mutants. The lac $Z$ mutants generated by both enzymes contained multiple sequence changes, consistent with the colorless plaque phenotype of most of the mutants. This and the high lac $\mathrm{Z}$ mutant frequencies indicate that both mammalian polymerases conduct low-fidelity synthesis when copying an undamaged DNA template. A variety of sequence changes were observed (summarized in Table 1).

\section{Base-substitution error rates and specificity}

The majority of pol $\eta$ errors are base substitutions (Figure 1). Those not located immediately adjacent to a flanking sequence change of any kind are categorized as single-base substitutions (Table 1, Figure 1(a)). The human and mouse enzymes generated 1560 and 122 single-base substitutions, respectively (Table 1). Given the total number of template nucleotides analyzed, the average single-base substitution error rates of human and mouse pol $\eta$ are $3.2 \times 10^{-2}$ and $2.2 \times 10^{-2}$, respectively $\dagger$.

Substitutions resulting from formation of all 12 mispairs were generated by both polymerases. The two enzymes have comparable error rates for these mispairs (Figure 2(a)), and statistical analysis (see the legend to Figure 2) indicates that their average error specificities are not sig- 
Table 1. Summary of sequence analysis of lacZ mutants generated by human and mouse DNA polymerase $\eta$

\begin{tabular}{|c|c|c|}
\hline & Human & Mouse \\
\hline Total lacZ mutants sequenced & 124 & 20 \\
\hline Bases sequenced per mutant & 407 & 275 \\
\hline Total bases sequenced ${ }^{a}$ & 50,468 & 5500 \\
\hline Single-base substitutions & 1560 & 122 \\
\hline Tandem base substitutions & 89 & 6 \\
\hline Substitution + deletion or addition & 34 & 0 \\
\hline Deletions: one base & 119 & 12 \\
\hline two or more bases & 87 & 7 \\
\hline Addition of one to three bases & 74 & 9 \\
\hline Complex deletion/addition & 9 & 0 \\
\hline
\end{tabular}

${ }^{a}$ The number of nucleotides actually sequenced is slightly less due to deletions partly counterbalanced by additions. For simplicity, and because the net number of nucleotides lost is small, we calculated error rates using the total number of nucleotides listed.

nificantly different. Error rates for the 12 mispairs vary over a considerable range for both human and mouse pol $\eta$. For example, the human pol $\eta$ error rate for the T.dGMP mispair is 70-fold higher than for the C.dCMP mispair (Figure 2(a), $6.3 \times 10^{-2}$ versus $9.3 \times 10^{-4}$ ). The substitution spectrum for human pol $\eta$ (Figure 1(a)) also reveals substantial sequencecontext effects on error rates. For example, the error rate for the T.dGMP mispair varies by 18 -fold, from $14 \times 10^{-2}$ at the template $\mathrm{T}$ at position -8 to $0.8 \times 10^{-2}$ at the immediately adjacent template $\mathrm{T}$ at position -7 or at positions +6 and +87 (Figure 1(a)). Sequence-dependent variations are observed for other substitutions (Figure 1(a)), yielding a range of error rates for each mispair (Figure 2(b)). The recovery of three base substitutions just upstream of $5^{\prime}$ end of the gap (Figure 1(a)) suggests that human pol $\eta$ can perform limited strand-displacement synthesis.

To further examine sequence-dependent biases in error rate, we analyzed the largest collection of single-base substitution events, the $\mathrm{T} \rightarrow \mathrm{C}$ substitutions shown in Figure 1(a). Considering only the bases immediately flanking these substitutions, we detected two- to threefold biases for misincorporation of dGMP opposite $\mathrm{T}$ when its $3^{\prime}$ neighbor is a $\mathrm{T} \cdot \mathrm{A}$ or A.T base-pair as compared to when it is a G.C or C.G base-pair (Figure 3). Given the large number of template $\mathrm{T}$ residues (99) and substitution events (665) considered, these biases are statistically significant (see the legend to Figure 3).

\section{Deletion and addition errors}

Both human and mouse pol $\eta$ also generated a variety of deletion and addition errors (Table 1 , Figure 4). Among these, the most frequent changes were single-base deletions (Table 1, 119 occurrences with human pol $\eta$ ) and single-base additions (64 occurrences with human pol $\eta$ ). The average error rates of the human enzyme for single-base deletions and additions are therefore $240 \times 10^{-5}$ and $130 \times 10^{-5}$, respectively. These rates are much higher than those of DNA polymerases in other families when measured using the same forward mutation assay (Table 2). The singlebase additions and deletions generated by pol $\eta$ are distributed throughout the template sequence (Figure 4(a)). From this distribution and the nucleotide composition of the template sequence, error rates for deletions and additions of specific nucleotides can be calculated. Thus, human pol $\eta$ has an average error rate of $150 \times 10^{-5}$ for deletion of one of the 204 non-iterated template nucleotides in the 407-nucleotide gap (Table 3). Interestingly, the average rates for deletions in homopolymeric runs of two to five nucleotides are only two- to threefold higher (albeit higher still at specific homopolymeric sites, Figure 4(a)). Also, the average single-base addition rates of human pol $\eta$ do not increase with increasing run length (Table 3 ). Moreover, 18 additions involved adding a nucleotide that was different from either of its neighbors. These error specificity data suggest that many pol $\eta$ deletion and addition errors may not be initiated by strand slippage (see below). In contrast to these

Table 2. Single-base deletion and addition error rates of human DNA pol $\eta$ compared to other DNA polymerases

\begin{tabular}{llcc}
\hline & & \multicolumn{2}{c}{ Error rate $\left(\times 10^{-5}\right)$} \\
\cline { 3 - 4 } DNA Polymerase & Family & Deletions & Additions \\
\hline Human pol $\eta$ (this study) & RAD30 & 240 & 130 \\
Human pol $\beta^{29}$ & Pol X & 13 & 0.2 \\
Human pol $\alpha^{36}$ & Pol B & 3.9 & 0.3 \\
HIV-1 RT T3,38 & RT & 3.8 & 3.2 \\
Klenow fragment ${ }^{39}$ & Pol A & 0.8 & 0.05 \\
\hline
\end{tabular}


(a)

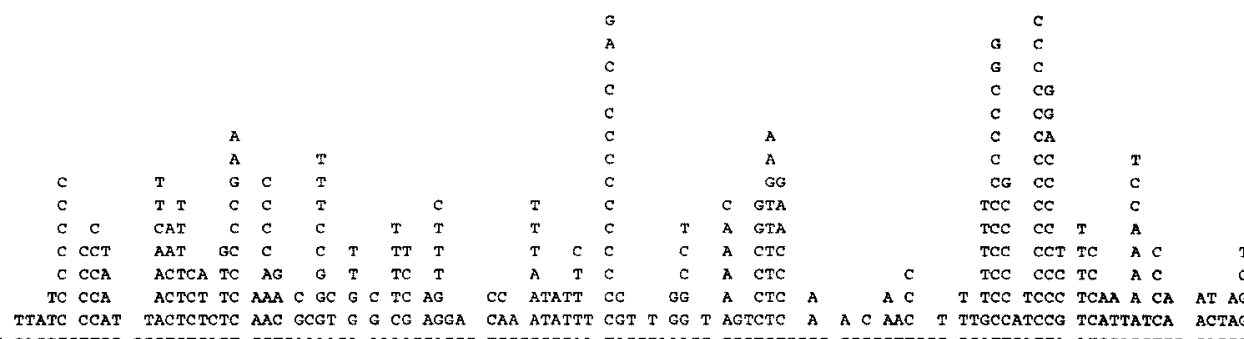

GAAGGGCAAT CAGCTGTTGC CCGTCTCACT GGTGAAAAGA AAAACCACCC TGGCGCCCAA TACGCAAACC GCCTCTCCCC GCGCGTTGGC CGATTCATTA ATGCAGCTGG CACGA

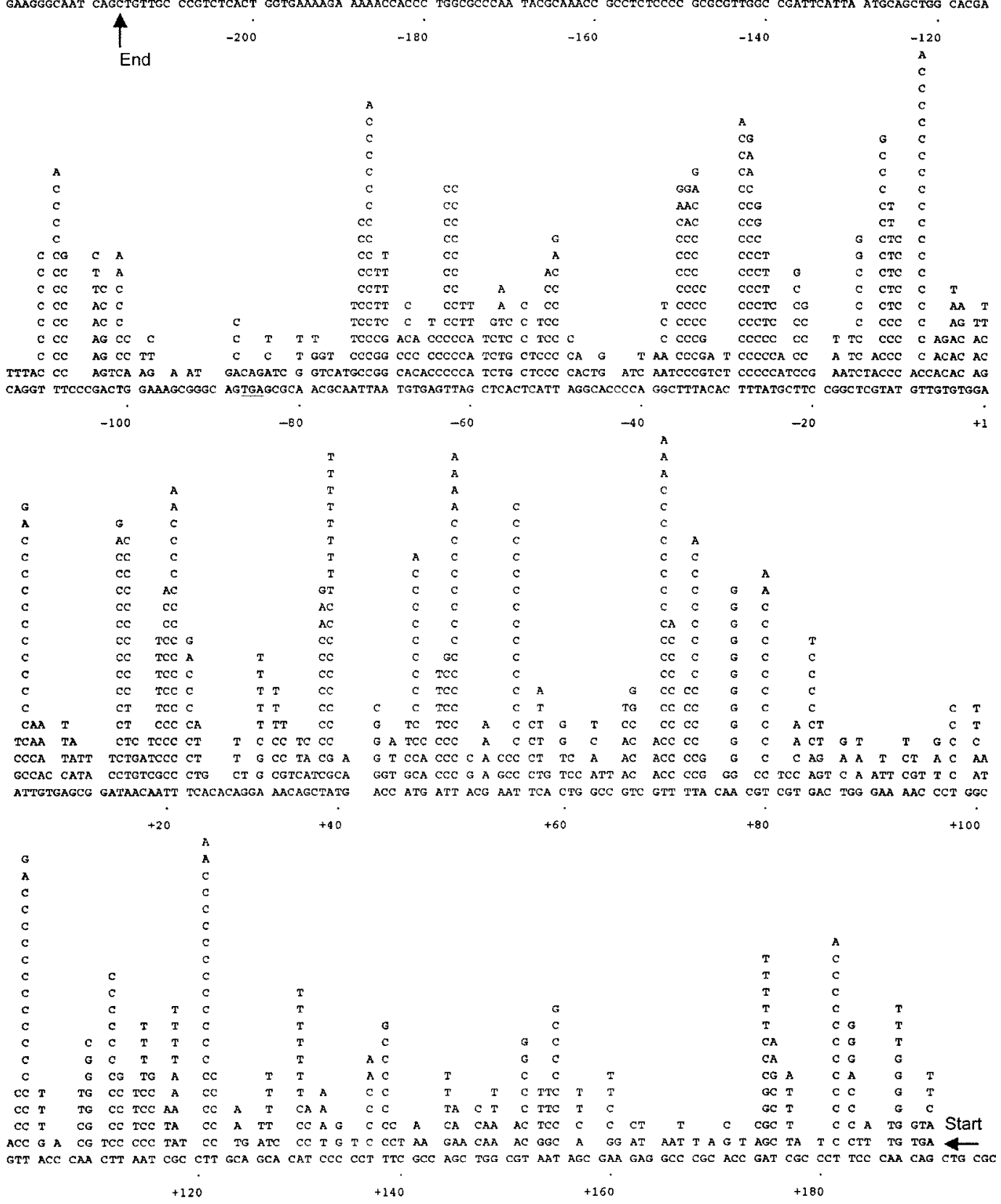

Figure 1 (legend opposite)

results with pol $\eta$, the single-base frameshift error rates of other DNA polymerases increase substantially with increasing run length, ${ }^{9}$ consistent with the strand slippage hypothesis ${ }^{10}$. As one example, the single-base deletion error rate of human pol $\beta$ in homopolymeric runs of four or five bases is 35fold higher than for deletion of non-iterated nucleotides (Table 3).

In addition to single-nucleotide frameshift errors, pol $\eta$ generated additions of two or three nucleo- 


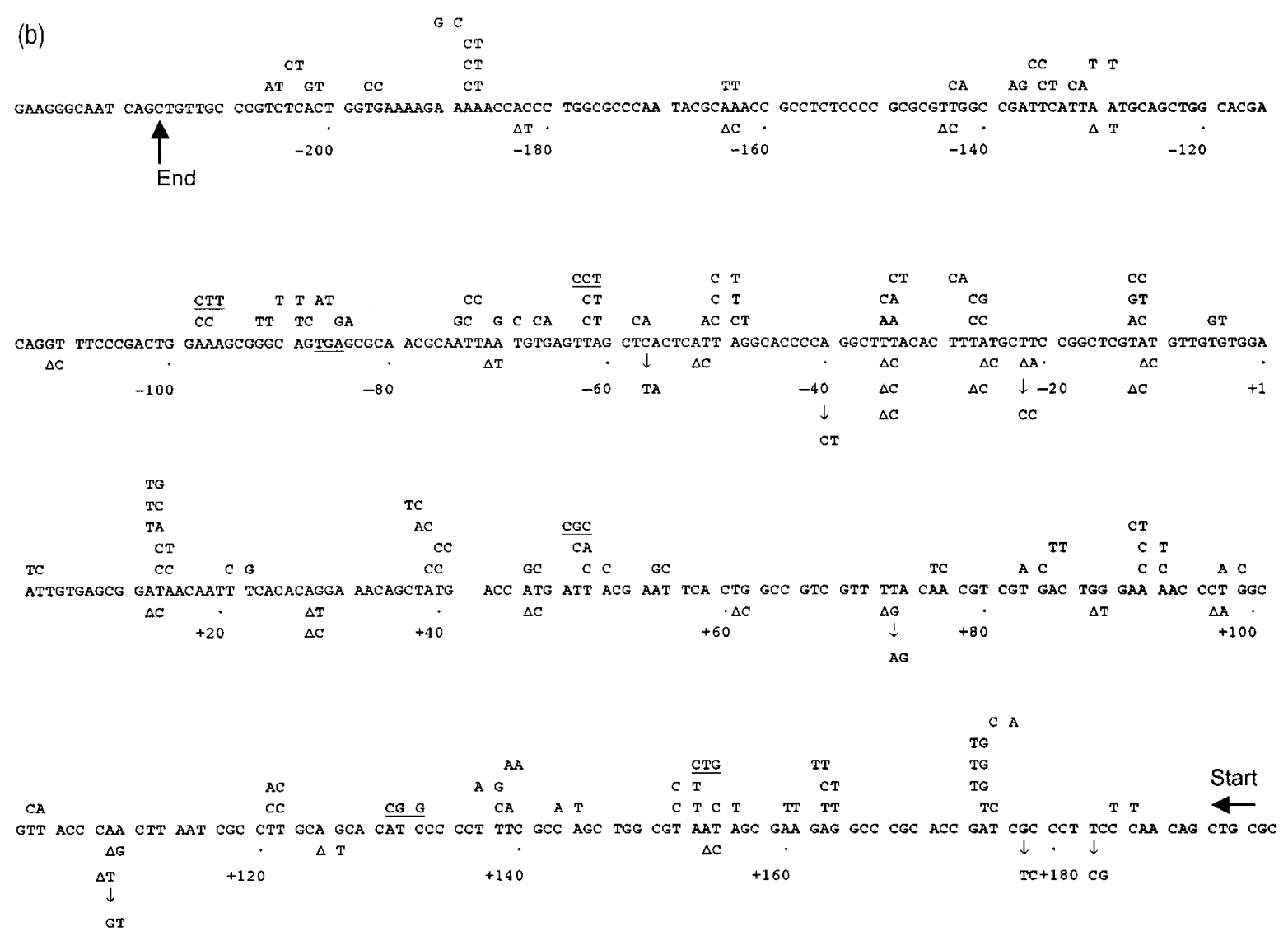

Figure 1. Spectra of base substitutions by human pol $\eta$. The 407 nucleotide template is shown as four lines of sequence. Nucleotide +1 is the first transcribed nucleotide of the lac $Z \alpha$-complementation gene in M13mp2 DNA. DNA synthesis begins with incorporation opposite template nucleotide +191 , marked with an arrow in the bottom line of sequence. The last single-stranded template nucleotide in the gap is at position -216 (arrow in top line of sequence). The termination codon for the carboxy-terminal end of the lacI gene in M13mp2 is underlined (nucleotides $-87,-86$ and -85 ) in the second line of template sequence. (a) Spectrum of single-base changes. (b) Spectrum of tandem changes. These include 84 tandem double-substitutions and five tandem triple-substitutions (underlined), shown as adjacent letters above the lines of sequence. Shown below the lines of sequence are 27 events where two nucleotides are replaced by one $(\Delta X)$ and seven events in which one nucleotide is replaced by two.

tides (Figure 4(a) and (b)), deletions of two to more than 100 nucleotides (Figure 4(a)-(c)), and a few complex addition/deletion mutations (Table 1). The locations of these errors suggest that some may involve strand slippage and homologous base-pairing (discussed below), but that others may arise by a different mechanism.

\section{Analysis of adjacent point mutations}

The errors made by pol $\eta$ included 84 tandem double-base substitutions. From these data, the calculated error rate for tandem double substitutions is $1.7 \times 10^{-3}$. These errors were widely distributed across the target sequence and involved various combinations of mispairs (Figure 1(b)). The extraordinary inaccuracy of human pol $\eta$ is further illustrated by the recovery of five tandem triplesubstitutions (underlined mutants in Figure 1(b)), and tandem events in which two template bases are replaced by one (substitution-deletion, 27 occurrences) and where one base is replaced by two (substitution-addition, seven occurrences).

\section{Extension kinetics for doubly mismatched primer termini}

The presence of 84 tandem double and five tandem triple-base substitutions in the error spectrum (Figure 1(b)) indicates that pol $\eta$ can extend primers containing multiple consecutive terminal mismatches. To investigate the efficiency of this reaction, we determined steady-state kinetic parameters for correct incorporation by human pol $\eta$ using two different, doubly mismatched termini. The results indicate that pol $\eta$ extends these substrates (Table 4, lines 7 and 8) with catalytic efficiencies that are only about tenfold lower than for the singly mismatched termini (lines 2, 3, 5 and 6) and 1000-fold lower than for extension of correct termini (lines 1 and 4). The efficiency of extension of these tandem double mismatches by pol $\eta$ is in 


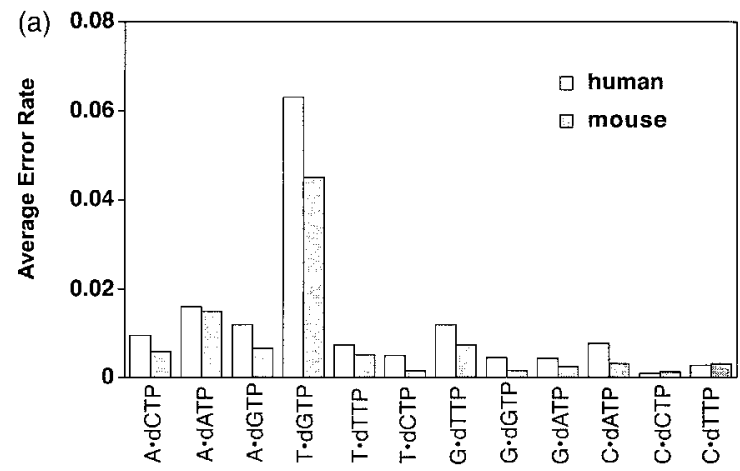

(b)

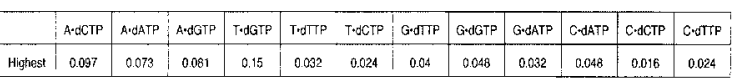

Figure 2. Error rates for 12 mispairs. (a) Average error rates for each of the 12 possible base base mispairs. (b) Listed is the highest site-specific error rate observed for each mispair, taken from the spectrum in Figure 1(a). In all cases, the lowest error rate was the same at $\leqslant 8 \times 10^{-3}$, because zero or one substitution was seen at (at least) one template site among 124 sequenced lacZ mutants. When a Monte Carlo modification of the Pearson $\chi^{2}$ test of spectra homogeneity was used to compare the human and mouse base substitution spectra, ${ }^{33,34}$ no significant difference was found $\left(P_{\mathrm{hg}}=0.97\right)$. A strong correlation (tau correlation coefficient $C C=0.43$, $P_{\text {cc }}<0.01^{35}$ ) was also observed when the two spectra were compared, as predicted for homogeneous spectra. Finally, various pairwise comparisons of rates indicate that the human and mouse data are similar $(C C=0.77$, $\left.P_{\mathrm{cc}}<0.01, P_{\mathrm{hg}}=0.67\right)$. These analyses suggest that the human and mouse enzymes do not differ significantly in error rates or error distribution.

fact similar to the efficiencies with which two other exonuclease-deficient replicative DNA polymerases extend single-base mismatches (Table 4, lines 9-12, from Mendelman et al. ${ }^{11}$ and Lewis et al. ${ }^{43}$ ).

\section{Discussion}

This study of the fidelity and error specificity of DNA polymerase $\eta$ has both biological and mechanistic implications. Like its human homo$\log { }^{6}$ mouse pol $\eta$ lacks an intrinsic proofreading exonucleolytic activity and has very low fidelity during copying of undamaged DNA. These facts and steady-state kinetic analysis showing that both yeast $^{8}$ and human pol $\eta$ misinsert nucleotides at rates that are high relative to many other DNA polymerases $^{6,7}$ indicate that low-fidelity DNA synthesis is a common property of DNA polymerase $\eta$. Following misinsertion, pol $\eta$ extends mismatched primer termini at rates that, while slower than for extension of matched termini, can nonetheless be substantial. ${ }^{12,13}$ In this study, promiscuity during mismatch extension is clearly

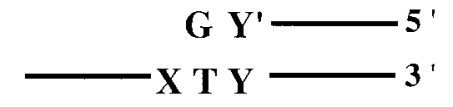

\begin{tabular}{|c|c|c|c|}
\hline $\mathbf{X}$ & $\begin{array}{l}\text { Mutations } \\
\text { per site }\end{array}$ & $\mathbf{Y} \cdot \mathbf{Y}^{\prime}$ & $\begin{array}{c}\text { Mutations } \\
\text { per site } \\
\end{array}$ \\
\hline A & 9.8 & $\mathbf{A} \cdot \mathbf{T}$ & 12 \\
\hline $\mathbf{T}$ & 8.8 & $\mathbf{T} \cdot \mathbf{A}$ & 11 \\
\hline C & 6.5 & $\mathrm{C} \cdot \mathrm{G}$ & 4.9 \\
\hline G & 6.4 & $G \cdot C$ & 4.0 \\
\hline
\end{tabular}

Figure 3. Sequence-context effects on formation of the $\mathrm{T}$.dGMP mispair by human pol $\eta$. The analysis is based on the location and number of $\mathrm{T} \rightarrow \mathrm{C}$ substitutions shown in Figure 1. We totaled the observed number of $\mathrm{T} \rightarrow \mathrm{C}$ substitutions $M(\underline{T Y})$ in all $\underline{T Y}$ sites, where the substituted site is underlined and $\mathrm{Y}^{-}=\mathrm{A}, \mathrm{T}, \mathrm{G}$ or $\mathrm{C}$. We calculated the number of TY dinucleotides, N(TY), in the target sequence. The expected number of $\mathrm{T} \rightarrow \mathrm{C}$ substitutions in TY sites, $E(\underline{T} Y)$, was estimated as:

$$
E(\underline{T Y})=[N(\mathrm{TY}) \times \Sigma M(\underline{T Y})] /[\Sigma N(\mathrm{TY})]
$$

The expected number of $\mathrm{T} \rightarrow \mathrm{C}$ substitutions $E(\mathrm{XT})$ $(\mathrm{X}=\mathrm{A}, \mathrm{T}, \mathrm{G}, \mathrm{C})$ in $\mathrm{XT}$ sites was estimated using the same equation. A chi-square test $\left(\chi^{2}\right)$ with three degrees of freedom was then used to evaluate the differences between the observed and expected number of $\mathrm{T} \rightarrow \mathrm{C}$ substitutions in TY and XT sites. For both XT and TY sites, the difference between the expected and the observed number of mutations was highly significant $(P<0.001)$. However, for the $\mathrm{XT}$ sites, the significance may partly reflect a larger number of WTW trinucleotides (28 sites) in comparison with STS (21 sites), WTS (21 sites) and STW trinucleotides (17 sites) in the target sequence (where $\mathrm{W}=\mathrm{A}$ or $\mathrm{T}$ and $\mathrm{S}=\mathrm{G}$ or $\mathrm{C}$ ).

illustrated by the frequent appearance of tandem double and triple-base substitutions in the human pol $\eta$ error spectrum (Figure 1(b)). It is indicated also by the ability of human pol $\eta$ to extend tandem double-mismatched termini at rates similar to those with which other polymerases extend single mismatches (Table 4). This property is interesting in light of our previous suggestion ${ }^{6}$ and evidence ${ }^{12}$ that slow mismatch extension, and possibly rapid dissociation as suggested by low processivity, may permit proofreading by an extrinsic exonuclease. This could reduce mutagenesis resulting from frequent misinsertions by pol $\eta$. However, promiscuous extension of certain mismatches by pol $\eta$ (e.g. T.dGMP, Table 4) may limit the opportunity for proofreading. In these instances, the cell may reduce the mutagenic potential of low-fidelity pol $\eta$ in at least two other ways. Strategic protein partnerships may target pol $\eta$ for use only when needed, e.g. for lesion bypass, or stable misincorporations by pol $\eta$ may be removed by DNA mismatch repair. 
Table 3. Relationship between homopolymeric run length and single-nucleotide deletion and addition error rates of human pol $\beta$ and pol $\eta$

\begin{tabular}{|c|c|c|c|c|}
\hline \multirow{3}{*}{$\begin{array}{l}\text { Homopolymeric run } \\
\text { length (nucleotides) }\end{array}$} & \multicolumn{4}{|c|}{ Error rate $\left(\times 10^{-5}\right)$} \\
\hline & \multicolumn{2}{|c|}{ Single-nucleotide deletions } & \multicolumn{2}{|c|}{ Single-nucleotide additions } \\
\hline & pol $\beta$ & pol $\eta$ & pol $\beta$ & pol $\eta$ \\
\hline One & 2.0 & 150 & $\leqslant 0.4$ & 180 \\
\hline Two & 9.3 & 270 & $\leqslant 0.7$ & 62 \\
\hline Three & 23 & 410 & $\leqslant 1.4$ & 57 \\
\hline Four and five & 70 & 320 & 2.3 & 160 \\
\hline
\end{tabular}

Both mouse and human pol $\eta$ generate all 12 single-base-base mispairs (Figure 2). Statistical analysis (see the legend to Figure 2) suggests that the human and mouse enzymes do not differ significantly in either error rate or error distribution. The error rate values in Figure 2 are substantially higher than those of most other DNA polymerases examined to date. The exceptions are African swine fever virus DNA polymerase and human pol $\mathrm{l}$, which are less accurate than pol $\eta$ for specific mismatches. ${ }^{14-17} \quad$ Structure-function studies ${ }^{5}$ suggest that the nucleotide selectivity of more accurate DNA polymerases in the Pol A, Pol X and RT families depends largely on base-pair geometry. ${ }^{5}$ The binding pockets of those polymerases are shaped to accommodate a correct Watson-Crick base-pair and to exclude an incorrect pair. The polymerase active site is suggested to exclude water and thus enhance nucleotide selectivity. ${ }^{18}$ In light of these ideas, the high basesubstitution error rates for pol $\eta$ (Figure 2) are remarkable, because they are similar to the rates of $1 / 10$ to $1 / 300$ predicted by free-energy differences between correct and incorrect base-pairs in duplex DNA in aqueous solution. ${ }^{19,20}$ This suggests that pol $\eta$ may have a more relaxed requirement for shape complementarity in order to achieve efficient catalysis during bypass of certain helix-distorting lesions, and that its active site may exclude water less effectively than more accurate polymerases.

Under these circumstances, incoming nucleotides may be stabilized by base-stacking interactions. With this possibility in mind, we performed an initial, simple analysis to determine if the nucleo-

Table 4. Kinetic constants for extension of various primer termini by human pol $\eta$

\begin{tabular}{|c|c|c|c|c|c|}
\hline $\begin{array}{l}\text { Template } \cdot \text { primer } \\
\text { (substrate) }\end{array}$ & Correct dNTP & $K_{\mathrm{m}}(\mu \mathrm{M})$ & $k_{\text {cat(app) }}\left(\min ^{-1}\right)$ & $\begin{array}{c}\left(k_{\text {cat }} / K_{\mathrm{m}}\right)_{\text {app }} \\
\left(\times 10^{3}\right)(\mu \mathrm{M} \cdot \mathrm{min})^{-1}\end{array}$ & $f_{\text {ext }}$ \\
\hline$G \cdot C(a)$ & dATP & $31 \pm 8.8$ & $13 \pm 4.4$ & $420 \pm 190$ & 1 \\
\hline $\mathrm{G} \cdot \mathrm{T}(\mathrm{a})$ & dATP & $730 \pm 160$ & $3.3 \pm 1.1$ & $4.5 \pm 1.8$ & 0.011 \\
\hline G.A (a) & dATP & $440 \pm 320$ & $1.6 \pm 0.9$ & $3.5 \pm 3.3$ & 0.008 \\
\hline $\mathrm{T} \cdot \mathrm{A}(\mathrm{b})$ & dGTP & $37 \pm 8.2$ & $11 \pm 0.0$ & $310 \pm 69$ & 1 \\
\hline $\mathrm{T} \cdot \mathrm{G}(\mathrm{b})$ & dGTP & $85 \pm 48$ & $8.4 \pm 0.9$ & $98 \pm 56$ & 0.32 \\
\hline $\mathrm{T} \cdot \mathrm{C}(\mathrm{b})$ & dGTP & $960 \pm 110$ & $3.2 \pm 1.8$ & $3.3 \pm 1.9$ & 0.011 \\
\hline $\mathrm{G} \cdot \mathrm{T} \& \mathrm{~T} \cdot \mathrm{C}(\mathrm{c})$ & dGTP & $1300 \pm 140$ & $0.44 \pm 0.18$ & $0.32 \pm 0.14$ & 0.001 \\
\hline $\mathrm{G} \cdot \mathrm{A} \& \mathrm{~T} \cdot \mathrm{C}(\mathrm{c})$ & dGTP & $1100 \pm 160$ & $0.30 \pm 0.08$ & $0.28 \pm 0.09$ & 0.0009 \\
\hline Pol $\alpha-G \cdot A^{11}$ & dGTP & & & & 0.0006 \\
\hline $\operatorname{Pol} \alpha-T \cdot C^{11}$ & dTTP & & & & 0.0002 \\
\hline HIV-1 RT-T. G G & dTTP & & & & 0.0009 \\
\hline HIV-1 RT-T. $C^{40}$ & dTTP & & & & 0.0002 \\
\hline
\end{tabular}

The results shown in the first six lines are from. ${ }^{15}$ Reactions were as described in Experimental Procedures, using the following substrates:

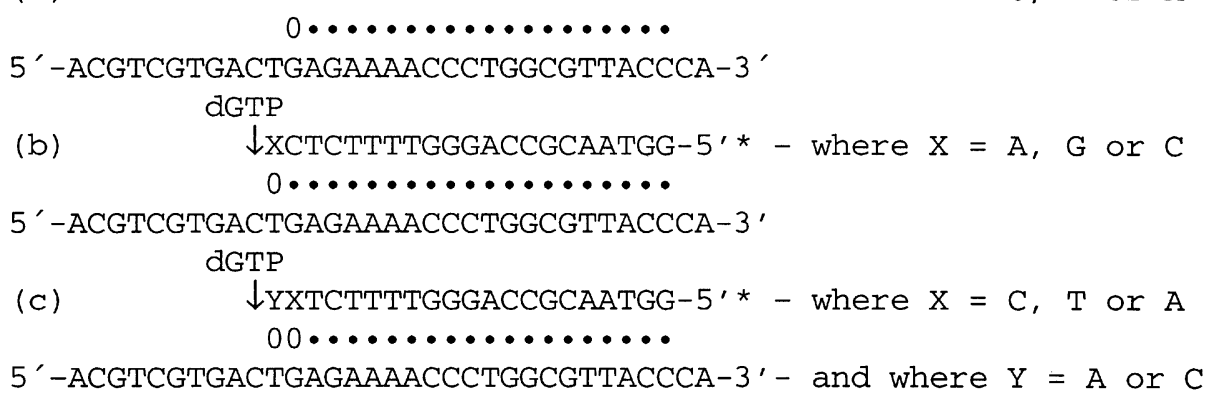


(a)

$$
\begin{array}{ccccccccc}
\multicolumn{9}{c}{\Delta \Delta \Delta \Delta \Delta} \\
\Delta \Delta & \Delta & \Delta \Delta & \Delta & & & \Delta & \Delta & \Delta
\end{array}
$$

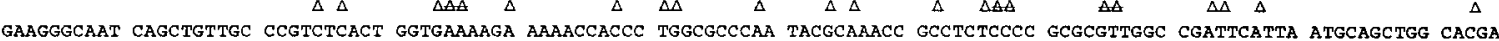

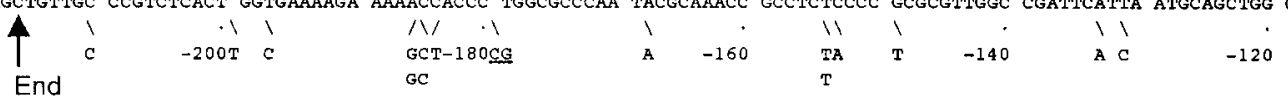

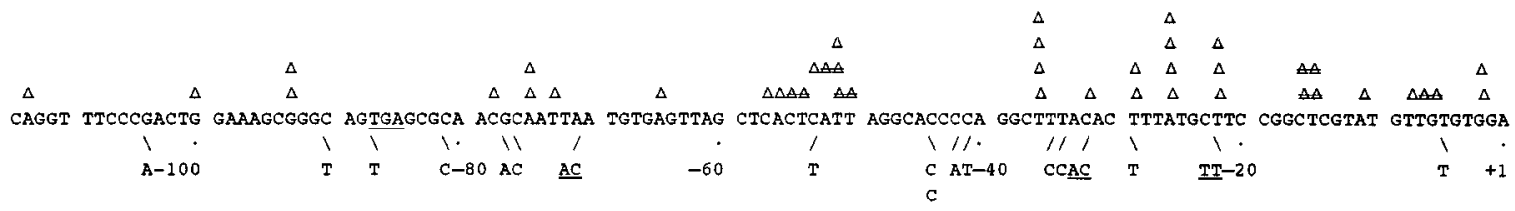

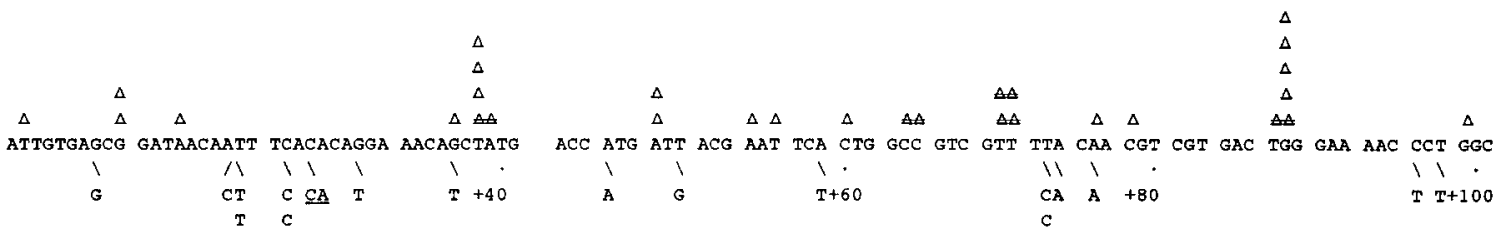

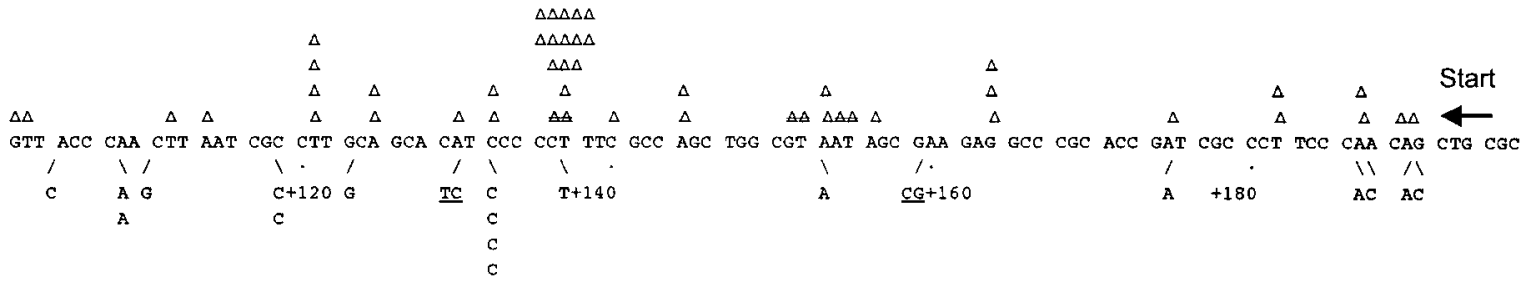

(b)
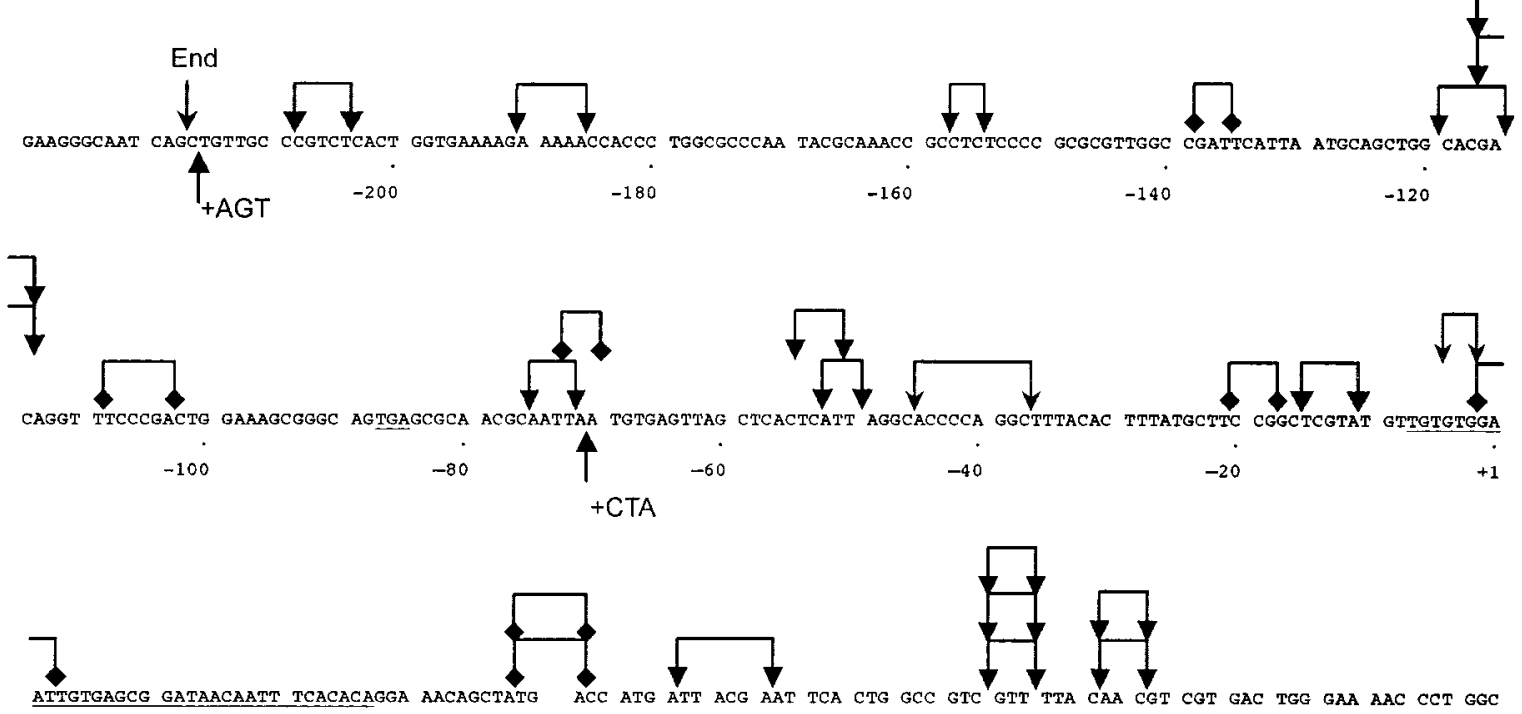

$+20$

$+40$

$+60$

$+80$

$+100$

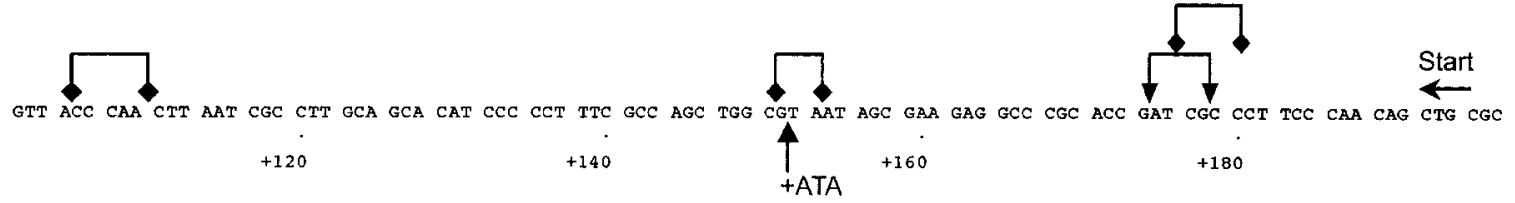

Figure 4 (legend opposite) 


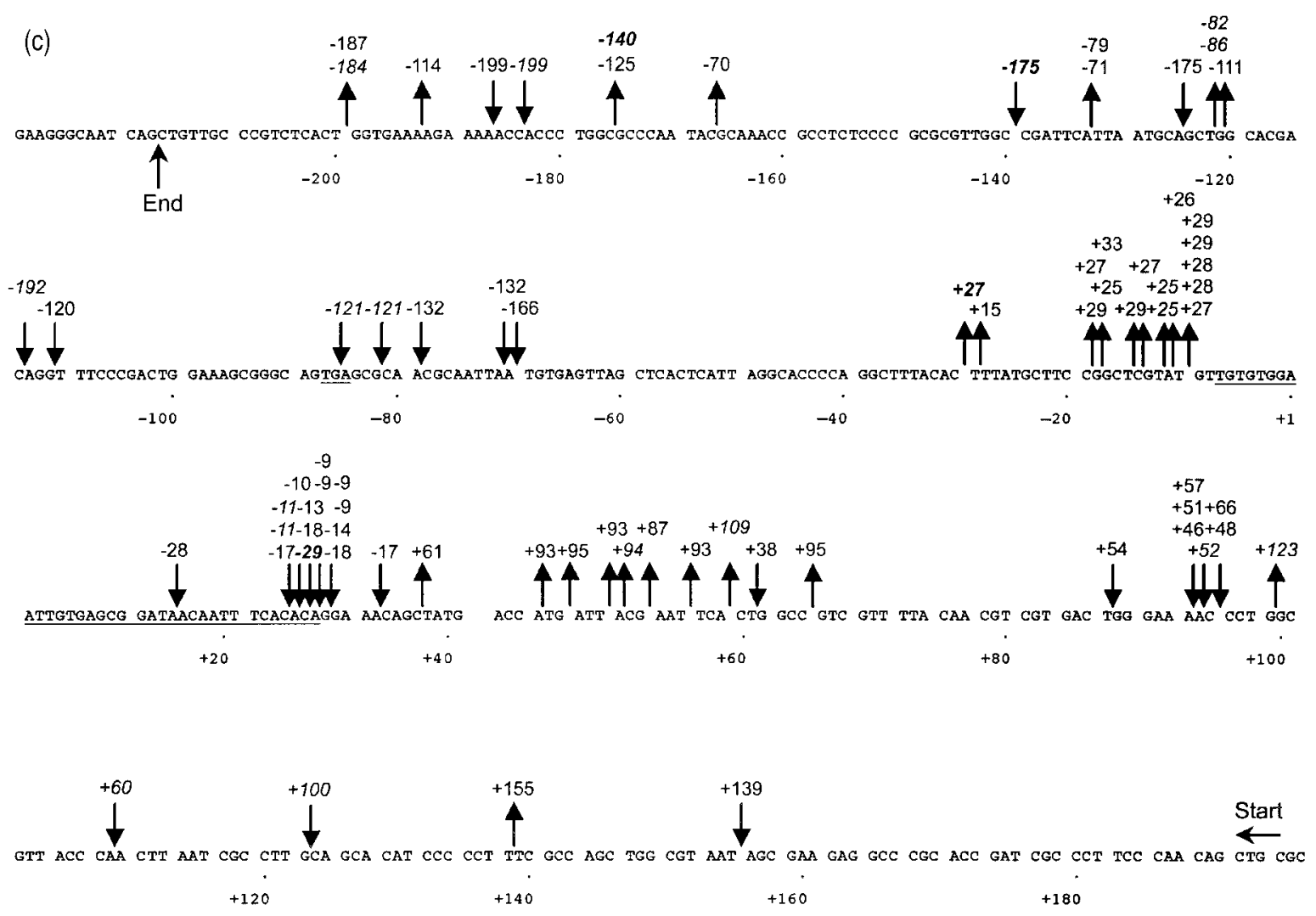

Figure 4. Spectra of deletion and addition errors by human pol $\eta$. The template sequence is the same as that shown in Figure 1. (a) Spectra of deletions (triangles) and additions (letters) of one or two nucleotides. The deletions are shown above each line of sequence, with single-base deletions as simple triangles and two-base deletions shown as adjacent triangles with lines. When deletions occur within repeat sequences, the exact base deleted is not known. In these cases, the triangle(s) are placed at the left border of the repetitive sequence. Additions are shown below each line of sequence, with single-base events indicated as simple letters and two-base additions shown as consecutive underlined letters. (b) Spectra of additions of three bases and deletions of three to nine bases. Three different threebase additions are shown as letters below the template sequence. Deletions are indicated by connected lines above the primary sequence. The two events designated with small arrowheads are deletions flanked by direct repeats. Events with the larger arrowheads are deletions whose endpoints are flanked by imperfect direct repeats, where a single misinsertion during synthesis of the first repeat would result in a perfect match upon pairing with the downstream repeat. The remaining events, designated with diamonds, are deletions flanked by sequences with little or no apparent homology. (c) The spectrum of 38 deletions of ten or more bases. Each deletion is defined by two arrows; one points down and the other points up. Each arrow is associated with a number indicating the nucleotide position at which the other endpoint for that deletion is located. As one example, a 17 nucleotide deletion was recovered whose endpoints are nucleotides +139 and +155 . This deletion is delineated by two arrows just above the lowest line of primary sequence. One arrow is associated with +139 , and it points down to indicate the deletion endpoint at nucleotide +155 . Similarly, the arrow pointing up at nucleotide +139 indicates the other endpoint and is associated with the number +155 . By using a similar pairing strategy, all 38 deletions can be identified with respect to the number of deleted nucleotides, the location of the endpoints and the nucleotides at the junction. Bold italicized numbers indicate deletions flanked by direct repeats. Non-bold italicized numbers indicate deletions whose endpoints are imperfect direct repeats, where a single misinsertion during copying of the first repeat results in a perfect match upon pairing with the downstream repeat. Plain numbers designate deletions flanked by sequences with no apparent homology.

tides immediately flanking the misincorporation site influenced the error rate. As typically seen with other DNA polymerases, pol $\eta$ error rates vary over a substantial range that depends on the mismatch composition, the mismatch symmetry (template versus incoming $\mathrm{dNTP}$ ), and on the nucleotide sequence surrounding the mismatch. Analysis of the distribution of the 665 observed
$\mathrm{T} \cdot \mathrm{dGMP}$ errors (Figure 3 ) indicates a higher error rate when the base-pair preceding the error is $\mathrm{T} \cdot \mathrm{A}$ or A.T than when it is G.C or C.G. This precludes the simple notion that the incoming incorrect dGTP is stabilized for incorporation more effectively when stacked with an adjacent purine rather than a pyrimidine. It may be that misincorporation of dGTP is favored when the primer terminus is 
slightly less stable (A.T rather than $G \cdot C$ ) and therefore more easily positioned for catalysis. The error spectrum shown in Figure 1 is the largest collection yet obtained for any DNA polymerase, providing a rich opportunity for a more detailed analysis of sequence-context effects on fidelity. Whatever the explanation for the non-random distribution of base-substitution errors, the data for human and mouse pol $\eta$ obtained here has already implied a novel and unanticipated biological role for this inaccurate polymerase. Statistical analysis of the pattern of substitutions in comparison to the specificity of somatic hypermutagenesis of immunoglobulin genes suggests that pol $\eta$ may participate in strand-specific hypermutation at A.T basepairs. ${ }^{21}$ If this hypothesis is correct, then the low fidelity with which pol $\eta$ copies undamaged DNA may provide an additional selective advantage to mammals beyond its role in translesion synthesis.

While most investigations of the fidelity of pol $\eta$ have focused on formation and extension of basebase mismatches, the present study clearly illustrates that both human and mouse pol $\eta$ also generate a variety of nucleotide deletion and addition errors (Tables 1-3, Figure 4). The error-specificity of human pol $\eta$ is remarkable in both the variety of errors observed (Figure 4) and in the rate at which they are generated. The average human pol $\eta$ error rates for single-nucleotide additions and deletions greatly exceed the frameshift error rates of most other DNA polymerases (Table 2). The only exception is human pol $\kappa$, whose single-base frameshift error rates ${ }^{22}$ approach those shown here for pol $\eta$. One logical explanation for formation of many of the frameshifts shown in Figure 4 is strand slippage involving repetitive sequences. ${ }^{10}$ A slippage mechanism ${ }^{9,23}$ may explain many of the frameshifts observed within homopolymeric sequences (Figure 4(a)) as well as those between distant directly repeated sequences (Figure 4(b) and (c)). The ability of pol $\eta$ to extend termini with one, two or even three mismatches may explain the high frequency and diverse nature of the deletions shown in Figure 4. Those deletions with limited homology at the endpoints, which includes most of those shown in Figure 4(b) and (c), may involve slippage between imperfect repeats. As proposed in earlier studies of single $e^{24-26}$ and multiple-base deletions, ${ }^{27}$ some of the deletions seen here may be initiated by a misinsertion that subsequently finds more favorable homology by relocating the primer to a downstream location. An additional factor contributing to primer relocation could be DNA secondary structure in the template. This is suggested by the clustering of deletion endpoints in the vicinity of a palindrome in the template (underlined sequence in Figure 4(c)) that can form a stable hairpin. Also note that the rate at which pol $\eta$ misinserts nucleotides is much higher than the deletion rate. Thus, misinsertion followed by primer relocation to create a misalinged templateprimer can readily explain the extraordinarily high rate of single-base frameshifts observed at non-iter- ated template positions (Table 3). Still another mechanism that could explain this class of errors is one in which a deletion intermediate is stabilized by stacking interactions with the incoming dNTP..$^{28-31}$

The fact that the single-base addition error rate of pol $\eta$ is much higher than those of other polymerases (Table 2), and that additions of two or three bases are observed (Figure 4) implies that pol $\eta$ has a remarkable capacity to perform synthesis using primers containing unpaired nucleotides. This is further illustrated by the fact that the difference in error rates for additions and deletions by pol $\eta$ is only twofold, whereas the difference is over tenfold with most other polymerases (Table 2). Moreover, many of the additions are not at repetitive sequence locations, suggesting that the additions may be initiated by a mechanism other than strand slippage. Hypothetically, this could involve misinsertion followed by primer relocation to create an unpaired nucleotide in the primer strand. However, more unusual models may be needed to account for the large number of additions wherein the nucleotide that is added differs from either of its neighbors (Figure 4(a)). Understanding the extraordinary infidelity and error-specificity of this remarkable DNA polymerase will undoubtedly be facilitated by structural information indicating how pol $\eta$ differs from other polymerases in its interactions with undamaged and damaged DNA at and upstream of the polymerase active site.

\section{Experimental Procedures}

\section{Materials}

All materials for the fidelity assay were from previously described sources ${ }^{41}$. C-terminal hexahistidinetagged human and mouse DNA polymerases $\eta$ were expressed in insect cells and purified as described.,42

\section{DNA synthesis reactions and analysis of fidelity}

Reactions $(25 \mu \mathrm{l})$ were performed as described, ${ }^{6,11}$ and contained $1.4 \mathrm{nM}$ M13mp2 DNA with a 407-nucleotide gap (from nucleotide -216 through +191 of the lac $Z$ gene), $40 \mathrm{mM}$ Tris- $\mathrm{HCl}$ ( $\mathrm{pH} 8.0), 10 \mathrm{mM} \mathrm{MgCl}{ }_{2}, 10 \mathrm{mM}$ dithiothreitol, $6.25 \mu \mathrm{g}$ of bovine serum albumin (BSA), $60 \mathrm{mM} \mathrm{KCl}, 2.5 \%$ (v/v) glycerol and $10 \mu \mathrm{M}, 100 \mu \mathrm{M}$ or $1 \mathrm{mM}$ dNTPs. Synthesis was initiated by adding 36 or $72 \mathrm{nM}$ pol $\eta$. Reactions were incubated at $37^{\circ} \mathrm{C}$ for one hour and terminated by adding EDTA to $15 \mathrm{mM}$. Agarose gel electrophoresis, performed as described, ${ }^{6}$ revealed that human and mouse pol $\eta$ filled the gap to completion. DNA products of the reactions with $72 \mathrm{nM}$ pol $\eta$ were examined for the frequency of lac $Z$ mutants as described. ${ }^{6}$ DNA from independent lacZ mutant phage was sequenced to identify the errors made by pol $\eta$ during gap-filling synthesis. Error rates were calculated as described above. 


\section{Mismatch extension kinetics}

Reactions $(25 \mu \mathrm{l})$ were performed as for fidelity measurements, except that they contained $200 \mathrm{nM}$ template primed at a 1.2:1 molar ratio with a $\left(5^{\prime}-{ }^{32} \mathrm{P}\right)$-labeled primer, $2 \mathrm{nM}$ pol $\eta$ and correct dATP or dGTP. The template-primers used are shown in Table 4. Duplicate determinations were performed with each template-primer using seven different concentration of the correct nucleotide. Aliquots were removed at two, four, six and eight minutes, and products were separated by electrophoresis in denaturing $16 \%(\mathrm{w} / \mathrm{v})$ polyacrylamide gels and quantified by phosphorimagery. Kinetic constants were derived as described. ${ }^{43}$

\section{Acknowledgments}

We thank Matthew Longley and Anna Bebenek for critical evaluation of the manuscript.

(C) 2001 US Government

\section{References}

1. Masutani, C., Kusumoto, R., Yamada, A., Dohmae, N., Yokoi, M. \& Yuasa, M., et al. (1999). The XPV (xeroderma pigmentosum variant) gene encodes human DNA polymerase $\eta$. Nature, 399, 700-704.

2. Johnson, R. E., Kondratick, C. M., Prakash, S. \& Prakash, L. (1999). hRAD30 mutations in the variant form of xeroderma pigmentosum. Science, 285, 263265.

3. Masutani, C., Araki, M., Yamada, A., Kusumoto, R., Nogimori, T., Maekawa, T. et al. (1999). Xeroderma pigmentosum variant $(\mathrm{XP}-\mathrm{V})$ correcting protein from HeLa cells has a thymine dimer bypass DNA polymerase activity. EMBO J. 18, 3491-3501.

4. Johnson, R. E., Prakash, S. \& Prakash, L. (1999). Efficient bypass of a thymine-thymine dimer by yeast DNA polymerase, Pol $\eta$. Science, 283, 1001-1004.

5. Kunkel, T. A. \& Bebenek, K. (2000). DNA replication fidelity. Annu. Rev. Biochem. 69, 497-529.

6. Matsuda, T., Bebenek, K., Masutani, C., Hanaoka, F. \& Kunkel, T. A. (2000). Low fidelity DNA synthesis by human DNA polymerase- $\eta$. Nature, 404, 10111013.

7. Johnson, R. E., Washington, M. T., Prakash, S. \& Prakash, L. (2000). Fidelity of human DNA polymerase $\eta$. J. Biol. Chem. 275, 7447-7450.

8. Washington, M. T., Johnson, R. E., Prakash, S. \& Prakash, L. (1999). Fidelity and processivity of Saccharomyces cerevisiae DNA polymerase $\eta$. J. Biol. Chem. 274, 36835-36838.

9. Bebenek, K. \& Kunkel, T. A. (2000). Streisinger revisited: DNA synthesis errors mediated by substrate misalignments. Cold Spring Harbor Symp. Quant. Biol. 65, 81-91.

10. Streisinger, G., Okada, Y., Emrich, J., Newton, J., Tsugita, A., Terzaghi, E. \& Inouye, M. (1966). Frameshift mutations and the genetic code. Cold Spring Harbor Symp. Quant. Biol. 31, 77-84.

11. Mendelman, L. V., Petruska, J. \& Goodman, M. F. (1990). Base mispair extension kinetics. Comparison of DNA polymerase alpha and reverse transcriptase. J. Biol. Chem. 265, 2338-2346.

12. Bebenek, K., Matsuda, T., Masutani, C., Hanaoka, F. \& Kunkel, T. A. (2001). Proofreading of DNA poly- merase $\eta$-dependent replication errors. J. Biol. Chem. 276, 2317-2320.

13. Washington, M. T., Johnson, R. E., Prakash, S. \& Prakash, L. (2001). Mismatch extension ability of yeast and human DNA polymerase $\eta$. J. Biol. Chem. 276, 2263-2266.

14. Showalter, A. K. \& Tsai, M.-D. (2001). A DNA polymerase with specificity for five base-pairs. J. Am. Chem. Soc. 123, 1776-1777.

15. Tissier, A., McDonald, J. P., Frank, E. G. \& Woodgate, R. (2000). pol ı, a remarkably error-prone human DNA polymerase. Genes Dev. 14, 1642-1650.

16. Zhang, Y., Yuan, F., Wu, X. \& Wang, Z. (2000). Preferential incorporation of $\mathrm{G}$ opposite template $\mathrm{T}$ by the low-fidelity human DNA polymerase 1. Mol. Cell. Biol. 20, 7099-7108.

17. Johnson, R. E., Washington, M. T., Haracska, L., Prakash, S. \& Prakash, L. (2000). Eukaryotic polymerases 1 and $\zeta$ act sequentially to bypass DNA lesions. Nature, 406, 1015-1019.

18. Petruska, J., Sowers, L. C. \& Goodman, M. F. (1986). Comparison of nucleotide interactions in water, proteins, and vacuum: model for DNA polymerase fidelity. Proc. Natl Acad. Sci. USA, 83, 1559-1562.

19. Raszka, M. \& Kaplan, N. O. (1972). Association by hydrogen bonding of mononucleotides in aqueous solution. Proc. Natl Acad. Sci. USA, 69, 2025-2029.

20. Mildvan, A. S. (1974). Mechanism of enzyme action. Annu. Rev. Biochem. 43, 357-399.

21. Rogozin, I. B., Pavlov, Y. I., Bebenek, K., Matsuda, T. \& Kunkel, T. A. (2001). Somatic mutation hotspots correlate with DNA polymerase $\eta$ error spectrum. Nature Immun. 2, 530-536.

22. Ohashi, E., Bebenek, K., Matsuda, T., Feaver, W. J., Gerlach, V. L., Friedberg, E. C. et al. (2000). Fidelity and processivity of DNA synthesis by DNA polymerase kappa, the product of the human DINB1 gene. J. Biol. Chem. 275, 39678-39684.

23. Kroutil, L. C. \& Kunkel, T. A. (1998). DNA replication errors involving strand misalignments. In Genetic Instabilities and Hereditary Neurological Diseases (Wells, R. D. \& Warren, S. T., eds), pp. 699-716, Academic Press, San Diego.

24. Kunkel, T. A. \& Soni, A. (1988). Mutagenesis by transient misalignment. J. Biol. Chem. 263, 1478414789.

25. Bebenek, K. \& Kunkel, T. A. (1990). Frameshift errors initiated by nucleotide misincorporation. Proc. Natl Acad. Sci. USA, 87, 4946-4950.

26. Bebenek, K., Roberts, J. D. \& Kunkel, T. A. (1992). The effects of dNTP pool imbalances on frameshift fidelity during DNA replication. J. Biol. Chem. 267, 3589-3596.

27. Cai, H., Yu, H., McEntee, K., Kunkel, T. A. \& Goodman, M. F. (1995). Purification and properties of wild-type and exonuclease-deficient DNA polymerase II from Escherichia coli. J. Biol. Chem. 270, 15327-15335.

28. Kunkel, T. A. (1986). Frameshift mutagenesis by eucaryotic DNA polymerases in vitro. J. Biol. Chem. 261, 13581-13587.

29. Osheroff, W. P., Beard, W. A., Yin, S., Wilson, S. H. \& Kunkel, T. A. (2000). Minor groove interactions at the DNA polymerase beta active site modulate single-base deletion error rates. J. Biol. Chem. 275, 28033-22808.

30. Efrati, E., Tocco, G., Eritja, R., Wilson, S. H. \& Goodman, M. F. (1997). Abasic translesion synthesis by DNA polymerase beta violates the "A-rule". 
Novel types of nucleotide incorporation by human DNA polymerase beta at an abasic lesion in different sequence contexts. J. Biol. Chem. 272, 2559-2569.

31. Hashim, M. F., Schnetz-Boutaud, N. \& Marnett, L. J. (1997). Replication of template-primers containing propanodeoxyguanosine by DNA polymerase beta. Induction of base-pair substitution and frameshift mutations by template slippage and deoxynucleoside triphosphate stabilization. J. Biol. Chem. 272, 20205-20212.

32. Umar, A., Boyer, J. C. \& Kunkel, T. A. (1994). DNA loop repair by human cell extracts. Science, 266, 814816.

33. Adams, W. T. \& Skopet, T. R. (1987). Statistical test for the comparison of samples from mutational spectra. J. Mol. Biol. 194, 391-396.

34. Piegorsch, W. W. \& Bailer, A. J. (1994). Statistical approaches for analyzing mutational spectra: some recommendations for categorical data. Genetics, 136, 403-416.

35. Babenko, V. N. \& Rogozin, I. B. (1999). Use of a rank correlation coefficient for comparing mutational spectra. Biofizika, 44, 632-638.

36. Roberts, J. D. \& Kunkel, T. A. (1996). Eukaryotic DNA replication fidelity. In DNA Replication in Eukaryotic Cells: Concepts, Enzymes and Systems (Pamphilis, M. D., ed.), pp. 217-247, Cold Spring Harbor Laboratory Press, Cold Spring Harbor, NY.

37. Bebenek, K., Abbotts, J., Roberts, J. D., Wilson, S. H. \& Kunkel, T. A. (1989). Specificity and mechanism of error-prone replication by human immunodeficiency virus-1 reverse transcriptase. J. Biol. Chem. 264, 16948-16956.

38. Bebenek, K., Abbotts, J., Wilson, S. H. \& Kunkel, T. A. (1993). Error-prone polymerization by HIV-1 reverse transcriptase: contribution of templateprimer misalignment, miscoding, and termination probability to mutational hot spots. J. Biol. Chem. 268, 10324-10334.

39. Bebenek, K., Joyce, C. M., Fitzgerald, M. P. \& Kunkel, T. A. (1990). The fidelity of DNA synthesis catalyzed by derivatives of Escherichia coli DNA polymerase I. J. Biol. Chem. 265, 13878-13887.

40. Yu, H. \& Goodman, M. F. (1992). Comparison of HIV-1 and avian virus reverse transcriptase fidelity on RNA and DNA templates. J. Biol. Chem. 267, 10888-10896.

41. Bebenek, K. \& Kunkel, T. A. (1995). Analyzing the fidelity of DNA polymerases. Methods Enzymol. 262, 217-232.

42. Masutani, C., Kusumoto, R., Iwai, S. \& Hanaoka, F. (2000). Mechanisms of accurate translesion synthesis by human DNA polymerase $\eta$. EMBO J. 19, 31003109.

43. Lewis, D. A., Bebenek, K., Beard, W. A., Wilson, S. H. \& Kunkel, T. A. (1999). Uniquely altered DNA replication fidelity conferred by an amino acid change in the nucleotide binding pocket of human immunodeficiency virus type 1 reverse transcriptase. J. Biol. Chem. 274, 32924-32930.

Edited by M. Yaniv

(Received 30 April 2001; received in revised form 11 July 2001; accepted 11 July 2001) 